\section{Control of Sonne Dysentery}

Many infectious diseases of childhood have undergone a spectacular decline, but transmissible diarrhoeas continue to remain a burden to sufferers, family doctors, and public health and education services. In England Sonne dysentery is common, and it is disappointing that outbreaks should still be so frequent and often inadequately managed. The disease can be readily diagnosed-bacteriological confirmation is possible in $90 \%$ of cases-and its method of transmission is common knowledge. In short it is largely preventable. Why then are outbreaks so difficult to control?

This unsatisfactory state of affairs may be influenced by beliefs that the disease is always so widespread throughout the general population as to defy control and that when outbreaks occur specific measures are unrewarding. Neither of these assumptions is true. Two recent papers by M. E. M. Thomas and $\mathrm{H}$. E. Tillett ${ }^{12}$ report the findings of the first long-term study of the epidemiology of Sonne dysentery in England and describe the measures of control found useful.

During their 16-year period of study, which was made in Enfield, Middlesex, Sonne dysentery accounted for no less than $10 \%$ of acute diarrhoeal illnesses investigated. But despite the large number of cases diagnosed during epidemic years the disease was never ubiquitous. Outbreaks of Sonne dysentery were virtually confined to situations in which large groups of children up to the age of 10 years were brought together in close contact. Thus they occurred only in primary schools and nurseries; there were no outbreaks in secondary schools. Moreover, only a small number of schools and nurseries were affected at any one time, and the problem was therefore manageable. The incidence was very low in adult men, and the greater exposure of mothers of young children was probably responsible for the higher case rate in adult women.

Sonne dysentery was not invariably a mild disease, for $4 \%$ of cases were admitted to hospital and $40 \%$ had blood or pus or both in the stools. Excretion of Shigella sonnei often lasted for less than two weeks, but about one in five children were still excreting it-some intermittently-one month after the onset of symptoms. Spread of infection to other members of the household was common. More than $40 \%$ of all children exposed in the home and $20 \%$ of adults became infected.

The authors' second report, which deals with the control of dysentery, indicates that outbreaks of Sonne dysentery in primary schools are best dealt with by the immediate exclusion of all children in whom recent diarrhoea is suspected. In Enfield the medical officer of health requested head teachers to co-operate in excluding suspects and reminded them to do so at regular intervals. Children excluded as suspects were not readmitted until bacteriologically clear.

The occurrence of an outbreak should be the signal for careful supervision of hygiene. After using the lavatory each child should be supervised while the hands are washed under running water and finally dipped in an appropriate disinfectant. Shared towels and stagnant basins of water are unsuitable. The importance of hand washing is such that in Enfield aides were employed as necessary to supervise toilet hygiene during school outbreaks.

The measures adequate for primary schools were not found sufficient for day nurseries. Experience in Enfield suggests that for the latter the best way to end an outbreak of dysentery quickly is to close the nursery for a few days for thorough cleaning. Every person in the nursery-children and staff-should then have adequate negative bacteriological clearance before being readmitted. At all times in- tending newcomers to a nursery should be screened for intestinal infection and parasites. Finally, it was considered important that health departments should be able to arrange for schools and nurseries to have direct access to a laboratory for the diagnosis of diarrhoeal disease. Taken together such relatively simple procedures could probably do much to reduce the incidence of Sonne dysentery.

1 Thomas, M. E. M., and Tillett, H. E., Fournal of Hygiene, 1973, 71, 373. 2 Thomas, M. E. M., and Tillett, H. E., Fournal of Hygiene, 1973, 71, 593.

\section{Radiological Aspects of Familial Medullary Carcinoma of the Thyroid}

Medullary carcinoma of the thyroid is a tumour of the parafollicular or C-cells, which produce calcitonin. These tumours comprise up to $10 \%$ of all thyroid cancers. They occur both sporadically and as part of a familial endocrine syndrome.

Recently K. D. Pearson and his colleagues ${ }^{1}$ have described the radiological features of the familial syndrome in a study of 21 members of a family with medullary carcinoma of the thyroid, adrenal phaeochromocytoma, and parathyroid hyperplasia. Tumour calcification was found in 7 of 19 patients studied. They usually had large, discrete calcified areas in the neck, which differed from the psammomatous calcification seen in papillary thyroid carcinoma. Similar calcification was observed in metastatic deposits in lymph nodes and the liver. Only one patient with a phaeochromocytoma had calcification in the adrenal tumour, which appeared as a thin calcified rim.

Metastases in the lungs were interstitial nodules with a fibrotic appearance. They later showed pulmonary hyperaeration, findings previously observed by $\mathrm{S}$. Wallace and colleagues. ${ }^{2}$ Metastatic nodules were most prominent in the mid-lung, unlike other diffuse thyroid pulmonary deposits, which are predominantly basal. Bone metastases were primarily osteolytic, but sclerotic and lytic-sclerotic deposits also occurred.

Chondrocalcinosis was observed on $x$-ray examination of the knees and wrists, and aspiration in one patient confirmed the presence of calcium pyrophosphate crystals. No radiological evidence of hyperparathyroidism was detected in the patients with chondrocalcinosis, but one had parathyroid hyperplasia at operation. Presumably some alteration in calcium metabolism associated with raised levels of calcitonin and parathyroid hormone was responsible for the cartilage calcification, since none of the other conditions known to be associated with "pseudo-gout" could be detected. Hyperparathyroidism due to parathyroid hyperplasia or adenoma may be associated with medullary carcinoma of the thyroid, but bony signs of parathyroid disease are uncommon.

Gastrointestinal symptoms are associated with medullary carcinoma of the thyroid, particularly diarrhoea, which may be the result of humoral factors secreted by the tumour. ${ }^{3}$ Some patients show radiological evidence of a megacolon 4 associa ed with ganglioneuromas of the bowel. Various arteriographic changes may be observed, including increased vascularity of the tumour, filling defects of varying size and 